\title{
Variational Principles for Multiwalled Carbon Nanotubes Undergoing Vibrations Based on Nonlocal Timoshenko Beam Theory
}

\author{
Ismail Kucuk, ${ }^{1}$ Ibrahim S. Sadek, ${ }^{1}$ and Sarp Adali ${ }^{2}$ \\ ${ }^{1}$ Department of Mathematics and Statistics, American University of Sharjah, Sharjah, \\ P.O. Box 26666, United Arab Emirates \\ ${ }^{2}$ School of Mechanical Engineering, University of KwaZulu-Natal, Durban, South Africa \\ Correspondence should be addressed to Ismail Kucuk, ikucuk@aus.edu
}

Received 28 October 2009; Accepted 2 February 2010

Academic Editor: Bohua Sun

Copyright (C) 2010 Ismail Kucuk et al. This is an open access article distributed under the Creative Commons Attribution License, which permits unrestricted use, distribution, and reproduction in any medium, provided the original work is properly cited.

\begin{abstract}
Variational principles are derived for multiwalled carbon nanotubes undergoing linear vibrations using the semi-inverse method with the governing equations based on nonlocal Timoshenko beam theory which takes small scale effects and shear deformation into account. Physical models based on the nonlocal theory approximate the nanoscale phenomenon more accurately than the local theories by taking small scale phenomenon into account. Variational formulation is used to derive the natural and geometric boundary conditions which give a set of coupled boundary conditions in the case of free boundaries which become uncoupled in the case of the local theory. Hamilton's principle applicable to this case is also given.
\end{abstract}

\section{Introduction}

In the present study, the variational principles and the natural boundary conditions are derived for multiwalled carbon nanotubes undergoing the transverse vibrations. The governing equations are based on the nonlocal theory of elasticity which gives more accurate results than local elastic theory by taking the small scale effects into account in the formulation. Variational principles applicable to the multiwalled nanotubes undergoing vibrations and the related boundary conditions were derived in [1] using a continuum model based on the nonlocal theory of EulerBernoulli beams. In the present study these results are extended to the case of multiwalled nanotubes undergoing transverse vibrations and the Hamilton's principle is derived.

The laws of continuum mechanics are known to be robust enough to treat intrinsically discrete objects only a few atoms in diameter [2]. Subsequent studies established the accuracy of continuum-based approaches to the mechanics of nanotubes. A study of the range of applicability of elastic beam theory to model nanotubes and nanorods was given in [3]. Beam models used to study the buckling and vibration behavior of carbon nanotubes (CNTs) mostly employed the Euler-Bernoulli or Timoshenko beam theories. The equation for an Euler-Bernoulli beam is expressed in terms of only one unknown, namely, the deflection of the beam, and neglects the effect of transverse shear deformation. However, for nanotubes with low length to diameter ratio, shear deformation can be substantial and can be taken into account using a Timoshenko beam model. In this case the governing equations have two dependent variables, namely, the slope and deflection of the beam and are able to predict the mechanical behavior of CNTs more accurately. Several studies on the buckling of nanotubes used these two beam models with the Euler-Bernoulli beam model used in [4-8] and the Timoshenko model in [9]. Vibration of multiwalled nanotubes was studied in [10] using a Timoshenko beam model.

However, small scale effects were not taken into account in these papers. The importance of size effects for nanosized 
structures has been emphasized in [11-15] where properties of nano materials have been obtained. Beam theories capable of taking the small scale effects into account are based on the nonlocal theory of elasticity which was developed in early seventies [16, 17]. The nonlocal theory was applied to the study of nanoscale Euler-Bernoulli and Timoshenko beams in a number of papers [18-27]. Variational formulations for various nonlocal beam models were given in [23]. The nonlocal Euler-Bernoulli and Timoshenko beam models were employed to investigate the buckling and vibration characteristics of CNTs in [28-33] and comparisons between the two models were given in these papers. These studies considered single and double-walled nanotubes involving mostly simply supported boundary conditions and analytical solutions of the differential equations. Variational formulations allow the implementation of approximate and numerical methods of solutions and facilitate the consideration of complicated boundary conditions, especially in the case of multiwalled nanotubes. Recently variational principles and the natural boundary conditions were derived for multiwalled CNTs modeled as nonlocal Euler-Bernoulli beams in a number of studies with CNTs subject to vibrations [1] and a buckling load [34] where the linear elastic theory was employed. Variational principles were derived for CNTs undergoing nonlinear vibrations in [35] using a local EulerBernoulli beam CNT model.

Present study differs from the studies [1, 34, 35] where CNTs were modeled as Euler-Bernoulli beams with the nonlocal elastic theory employed in the case of CTNs undergoing linear vibrations [1] and buckling [34]. In the case of CTNs undergoing nonlinear vibrations again EulerBernoulli beam was used as a model which was based on the local elastic theory [35]. Euler-Bernoulli models are mostly applicable to nanotubes with a large length to diameter ratio and become inaccurate as the nanotubes become shorter. In the present study multiwalled CNTs are modeled as nonlocal Timoshenko beams which are applicable to nanotubes with a small length to diameter ratio and as such give accurate solutions for short CNTs [9, 10, 23-25].

The approach used in the present study to derive the variational principles is the semi-inverse method developed by $\mathrm{He}[36,37]$. Several examples of variational principles for systems of differential equations obtained by this method can be found in [38-42] and in the references therein. In the present study first the coupled differential equations governing the vibrations of multiwalled nanotubes based on nonlocal Timoshenko beam theory are given. Next a trial variational functional is formulated and a set of integrability conditions is derived which ensure that a classical variational principle can be obtained for the problem. Finally the variational principle and the Hamilton's principle are obtained by the semi-inverse method and natural and geometric boundary conditions are derived.

\section{Multiwalled Carbon Nanotubes}

A multiwalled carbon nanotube of length $L$ consisting of $n$ nanotubes of cylindrical shape is considered. It lies on a
Winkler foundation of modulus $k$ and is subject to an axial stress $\sigma_{x}$ which can be tensile or compressive in which case $\sigma_{x}$ is less than the critical buckling load. We introduce a difference operator defined as

$$
\Delta w_{i j}=w_{i}-w_{j}
$$

where $w_{i}$ and $w_{j}$ are the deflections of the $i$ th and $j$ th nanotubes. The differential equations governing the vibrations of multiwalled nanotubes based on the nonlocal Timoshenko beam theory can be expressed as $[10,24]$

$$
\begin{aligned}
D_{a 1}\left(w_{1}, \varphi_{1}, w_{2}\right)= & L_{a 1}\left(w_{1}, \varphi_{1}\right)-c_{12} \Delta w_{21} \\
& +\eta^{2} c_{12} \frac{\partial^{2} \Delta w_{21}}{\partial x^{2}}=0 \\
D_{b 1}\left(w_{1}, \varphi_{1}\right)= & L_{b 1}\left(w_{1}, \varphi_{1}\right)=0 \\
D_{a 2}\left(w_{1}, w_{2}, \varphi_{2}, w_{3}\right)= & L_{a 2}\left(w_{2}, \varphi_{2}\right)+c_{12} \Delta w_{21}-c_{23} \Delta w_{32} \\
& +\eta^{2}\left(-c_{12} \frac{\partial^{2} \Delta w_{21}}{\partial x^{2}}+c_{23} \frac{\partial^{2} \Delta w_{32}}{\partial x^{2}}\right) \\
= & 0, \\
D_{a n}\left(w_{n-1}, w_{n}, \varphi_{n}\right)= & L_{a n}\left(w_{n}, \varphi_{n}\right)+c_{(n-1) n} \Delta w_{n(n-1)} \\
& -\eta^{2} c_{(n-1) n} \frac{\partial^{2} \Delta w_{n(n-1)}}{\partial x^{2}}=f(x, t) \\
D_{b 2}\left(w_{2}, \varphi_{2}\right)= & L_{b 2}\left(w_{2}, \varphi_{2}\right)=0, \\
& +\eta^{2} c_{i(i+1)} \frac{\partial^{2} \Delta w_{(i+1) i}}{\partial x^{2}}=0 \\
D_{b i}\left(w_{i}, \varphi_{i}\right)=L_{b i}\left(w_{i}, \varphi_{i}\right)=0 & \\
D_{a i}\left(w_{i-1}, w_{i}, \varphi_{i}, w_{i+1}\right)= & L_{a i}\left(w_{i}, \varphi_{i}\right)+c_{(i-1) i} \Delta w_{i(i-1)} \\
& -c_{i(i+1)} \Delta w_{(i+1) i} \\
& -\eta^{2} c_{(i-1) i} \frac{\partial^{2} \Delta w_{i(i-1)}}{\partial x^{2}} \\
& \\
& \\
&
\end{aligned}
$$

$$
D_{b n}\left(w_{n}, \varphi_{n}\right)=L_{b n}\left(w_{n}, \varphi_{n}\right)=0,
$$


where $\varphi_{i}$ is the angle of rotation and the operators $L_{a i}\left(w_{i}, \varphi_{i}\right)$ and $L_{b i}\left(w_{i}, \varphi_{i}\right)$ are given by

$$
\begin{aligned}
L_{a i}\left(w_{i}, \varphi_{i}\right)= & \rho A_{i} \frac{\partial^{2} w_{i}}{\partial t^{2}}-\rho A_{i} \eta^{2} \frac{\partial^{4} w_{i}}{\partial t^{2} \partial x^{2}}+\kappa G A_{i} \frac{\partial}{\partial x}\left(\varphi_{i}-\frac{\partial w_{i}}{\partial x}\right) \\
& +A_{i} \sigma_{x} \frac{\partial^{2} w_{i}}{\partial x^{2}}-A_{i} \sigma_{x} \eta^{2} \frac{\partial^{4} w_{i}}{\partial x^{4}}+\delta_{i n}\left(k w_{n}-k \eta^{2} \frac{\partial^{2} w_{n}}{\partial x^{2}}\right)
\end{aligned}
$$

$$
\begin{aligned}
& L_{b i}\left(w_{i}, \varphi_{i}\right) \\
& \quad=\rho I_{i} \frac{\partial^{2} \varphi_{i}}{\partial t^{2}}-\rho I_{i} \eta^{2} \frac{\partial^{4} \varphi_{i}}{\partial t^{2} \partial x^{2}}+\kappa G A_{i}\left(\varphi_{i}-\frac{\partial w_{i}}{\partial x}\right)-E I_{i} \frac{\partial^{2} \varphi_{i}}{\partial x^{2}}
\end{aligned}
$$

where the index $i=1,2, \ldots, n$ refers to the order of the nanotubes with the innermost nanotube indicated by $i=1$ and the outermost nanotube by $i=n$ with $0 \leq x \leq L$. In (8) $f(x, t)$ is a forcing function, and in (10) $\delta_{i n}$ is the Kronecker's delta with $\delta_{i n}=0$ for $i \neq n$ and $\delta_{n n}=1$. In (10) and (11), $E$ is the Young's modulus, $G$ is the shear modulus, $\kappa$ is the shear correction factor, $I_{i}$ is the moment of inertia, $A_{i}$ is the cross-sectional area of the $i$ th nanotube and $\rho$ is the density. The coefficient $c_{(i-1) i}$ is the interaction coefficient of van der Waals forces between the $(i-1)^{\text {th }}$ and $i$ th nanotubes with $i=2, \ldots, n[7-10,28]$. The parameter $\eta=e_{0} a$ appears in the nonlocal theory of beams and helps define the small scale effects accurately where $e_{0}$ is a constant for adjusting the model by experimental results and $a$ is an internal characteristic length [17-26].

\section{Variational Formulation}

According to the semi-inverse method [36, 37], a variational trial-functional $V\left(w_{i}, \varphi_{i}\right)$ can be constructed as follows with the motion taking place between the initial time $t_{1}$ and the final time $t_{2}$

$$
\begin{aligned}
V\left(w_{i}, \varphi_{i}\right)= & V_{1}\left(w_{1}, \varphi_{1}, w_{2}\right)+V_{2}\left(w_{1}, w_{2}, \varphi_{2}, w_{3}\right) \\
& +\cdots+V_{n-1}\left(w_{n-2}, w_{n-1}, \varphi_{n-1}, w_{n}\right) \\
& +V_{n}\left(w_{n-1}, w_{n}, \varphi_{n}\right)
\end{aligned}
$$

where

$$
\begin{array}{r}
V_{1}\left(w_{1}, \varphi_{1}, w_{2}\right)=U_{1}\left(w_{1}, \varphi_{1}\right)+\int_{t_{1}}^{t_{2}} \int_{0}^{L} F_{1}\left(w_{1}, w_{2}\right) d x d t, \\
V_{2}\left(w_{1}, w_{2}, \varphi_{2}, w_{3}\right)=U_{2}\left(w_{2}, \varphi_{2}\right)+\int_{t_{1}}^{t_{2}} \int_{0}^{L} F_{2}\left(w_{1}, w_{2}, w_{3}\right) d x d t, \\
V_{i}\left(w_{i-1}, w_{i}, \varphi_{i}, w_{i+1}\right)=U_{i}\left(w_{i}, \varphi_{i}\right)+\int_{t_{1}}^{t_{2}} \int_{0}^{L} F_{i}\left(w_{i-1}, w_{i}, w_{i+1}\right) d x d t \\
\text { for } i=3,4, \ldots, n-1,
\end{array}
$$

$$
\begin{aligned}
V_{n}\left(w_{n-1}, w_{n}, \varphi_{n}\right)= & U_{n}\left(w_{n}, \varphi_{n}\right) \\
& +\frac{1}{2} \int_{t_{1}}^{t_{2}} \int_{0}^{L}\left(k w_{n}^{2}+k \eta^{2}\left(\frac{\partial w_{n}}{\partial x}\right)^{2}\right) d x d t \\
& +\int_{t_{1}}^{t_{2}} \int_{0}^{L}\left(-f w_{n}+F_{n}\left(w_{n-1}, w_{n}\right)\right) d x d t
\end{aligned}
$$

with $U_{i}\left(w_{i}, \varphi_{i}\right)$ given by

$$
\begin{gathered}
U_{i}\left(w_{i}, \varphi_{i}\right) \\
=\frac{1}{2} \int_{t_{1}}^{t_{2}} \int_{0}^{L}\left(\kappa G A_{i}\left(\varphi_{i}-\frac{\partial w_{i}}{\partial x}\right)^{2}+E I_{i}\left(\frac{\partial \varphi_{i}}{\partial x}\right)^{2}\right. \\
\left.-A_{i} \sigma_{x}\left(\frac{\partial w_{i}}{\partial x}\right)^{2}-A_{i} \sigma_{x} \eta^{2}\left(\frac{\partial^{2} w_{i}}{\partial x^{2}}\right)^{2}\right) d x d t \\
+\frac{1}{2} \int_{t_{1}}^{t_{2}} \int_{0}^{L}\left(-\rho A_{i}\left(\frac{\partial w_{i}}{\partial t}\right)^{2}-\rho A_{i} \eta^{2}\left(\frac{\partial^{2} w_{i}}{\partial t \partial x}\right)^{2}\right. \\
\left.-\rho I_{i}\left(\frac{\partial \varphi_{i}}{\partial t}\right)^{2}-\rho I_{i} \eta^{2}\left(\frac{\partial^{2} \varphi_{i}}{\partial t \partial x}\right)^{2}\right) d x d t
\end{gathered}
$$

where $i=1,2, \ldots, n$ and $F_{i}\left(w_{i-1}, w_{i}, w_{i+1}\right)$ denotes the unknown functions of $w_{i}$ and its derivatives to be determined such that the differential equations (2)-(9) correspond to the Euler-Lagrange equations of the variational functional (12). These equations are given by

$$
\begin{aligned}
& L_{a 1}\left(w_{1}, \varphi_{1}\right)+\sum_{j=1}^{2} \frac{\delta F_{j}}{\delta w_{1}} \\
& =L_{a 1}\left(w_{1}, \varphi_{1}\right) \\
& \quad+\sum_{j=1}^{2}\left[\frac{\partial F_{j}}{\partial w_{1}}-\frac{\partial}{\partial x}\left(\frac{\partial F_{j}}{\partial w_{1 x}}\right)-\frac{\partial}{\partial t}\left(\frac{\partial F_{j}}{\partial w_{1 t}}\right)+\cdots\right]=0, \\
& L_{a 2}\left(w_{2}, \varphi_{2}\right)+\sum_{j=1}^{3} \frac{\delta F_{j}}{\delta w_{2}} \\
& =L_{a 2}\left(w_{2}, \varphi_{2}\right) \\
& \quad+\sum_{j=1}^{3}\left[\frac{\partial F_{j}}{\partial w_{2}}-\frac{\partial}{\partial x}\left(\frac{\partial F_{j}}{\partial w_{2 x}}\right)-\frac{\partial}{\partial t}\left(\frac{\partial F_{j}}{\partial w_{1 t}}\right)+\cdots\right]=0, \\
& L_{a i}\left(w_{i}, \varphi_{i}\right)+\sum_{j=i-1}^{i+1} \frac{\delta F_{j}}{\delta w_{i}} \\
& =L_{a i}\left(w_{i}, \varphi_{i}\right) \\
& \quad+\sum_{j=i-1}^{i+1}\left[\frac{\partial F_{j}}{\partial w_{i}}-\frac{\partial}{\partial x}\left(\frac{\partial F_{j}}{\partial w_{i x}}\right)-\frac{\partial}{\partial t}\left(\frac{\partial F_{j}}{\partial w_{i t}}\right)+\cdots\right]=0, \\
& \quad \text { for } i=3,4, \ldots, n-1,
\end{aligned}
$$




$$
\begin{aligned}
& L_{a n}\left(w_{n}, \varphi_{n}\right)+\sum_{j=n-1}^{n} \frac{\delta F_{j}}{\delta w_{n}} \\
& =L_{a n}\left(w_{n}, \varphi_{n}\right) \\
& \quad+\sum_{j=n-1}^{n}\left[\frac{\partial F_{j}}{\partial w_{n}}-\frac{\partial}{\partial x}\left(\frac{\partial F_{j}}{\partial w_{n x}}\right)-\frac{\partial}{\partial t}\left(\frac{\partial F_{j}}{\partial w_{n t}}\right)+\cdots\right]=0, \\
& L_{b i}\left(w_{i}, \varphi_{i}\right)=0 \text { for } i=1,2, \ldots, n,
\end{aligned}
$$

where the subscripts $x$ and $t$ denote differentiation with respect to $x$ and $t$, and the variational derivative $\delta F_{i} / \delta w_{i}$ is defined as $[36,37]$

$$
\begin{aligned}
\frac{\delta F_{i}}{\delta w_{i}}= & \frac{\partial F_{i}}{\partial w_{i}}-\frac{\partial}{\partial x}\left(\frac{\partial F_{i}}{\partial w_{i x}}\right)-\frac{\partial}{\partial x}\left(\frac{\partial F_{i}}{\partial w_{i t}}\right)+\frac{\partial^{2}}{\partial x^{2}}\left(\frac{\partial F_{i}}{\partial w_{i x x}}\right) \\
& +\frac{\partial^{2}}{\partial x \partial t}\left(\frac{\partial F_{i}}{\partial w_{i x t}}\right) \cdots-\frac{\partial}{\partial t}\left(\frac{\partial F_{i}}{\partial w_{i t}}\right)+\frac{\partial^{2}}{\partial t^{2}}\left(\frac{\partial F_{i}}{\partial w_{i t t}}\right)+\cdots
\end{aligned}
$$

Comparison of (15) with (2)-(9) indicates that the following equations have to be satisfied for Euler-Lagrange equations to represent the governing (2)-(9)

$$
\begin{aligned}
\sum_{j=1}^{2} \frac{\delta F_{j}}{\delta w_{1}}= & -c_{12} \Delta w_{21}+\eta^{2} c_{12} \frac{\partial^{2} \Delta w_{21}}{\partial x^{2}} \\
\sum_{j=1}^{3} \frac{\delta F_{j}}{\delta w_{2}}= & c_{12} \Delta w_{21}-c_{23} \Delta w_{32}-\eta^{2} c_{12} \frac{\partial^{2} \Delta w_{21}}{\partial x^{2}} \\
& +\eta^{2} c_{23} \frac{\partial^{2} \Delta w_{32}}{\partial x^{2}}, \\
\sum_{j=i-1}^{i+1} \frac{\delta F_{j}}{\delta w_{i}}= & c_{(i-1) i} \Delta w_{i(i-1)}-c_{i(i+1)} \Delta w_{(i+1) i} \\
& -\eta^{2} c_{(i-1) i} \frac{\partial^{2} \Delta w_{i(i-1)}}{\partial x^{2}}+\eta^{2} c_{i(i+1)} \frac{\partial^{2} \Delta w_{(i+1) i}}{\partial x^{2}} \\
\sum_{j=n-1}^{n} \frac{\delta F_{j}}{\delta w_{n}}= & c_{(n-1) n} \Delta w_{n(n-1)}-\eta^{2} c_{(n-1) n} \frac{\partial^{2} \Delta w_{n(n-1)}}{\partial x^{2}} .
\end{aligned}
$$

Integrability relations between these equations can be obtained by noting that

$$
\begin{aligned}
& \left(\frac{\partial}{\partial w_{2}}+\frac{\partial}{\partial w_{2 x x}}\right) \sum_{j=1}^{2} \frac{\delta F_{j}}{\delta w_{1}}=-c_{12}+\eta^{2} c_{12}, \\
& \left(\frac{\partial}{\partial w_{1}}+\frac{\partial}{\partial w_{1 x x}}\right) \sum_{j=1}^{3} \frac{\delta F_{j}}{\delta w_{2}}=-c_{12}+\eta^{2} c_{12},
\end{aligned}
$$

$$
\begin{aligned}
& \left(\frac{\partial}{\partial w_{i+1}}+\frac{\partial}{\partial w_{(i+1) x x}}\right) \sum_{j=i-1}^{i+1} \frac{\delta F_{j}}{\delta w_{i}}=-c_{i(i+1)}+\eta^{2} c_{i(i+1)} \\
& \left(\frac{\partial}{\partial w_{i}}+\frac{\partial}{\partial w_{i x x}}\right) \sum_{j=i}^{i+2} \frac{\delta F_{j}}{\delta w_{i+1}}=-c_{i(i+1)}+\eta^{2} c_{i(i+1)} \\
& \left(\frac{\partial}{\partial w_{n}}+\frac{\partial}{\partial w_{n x x}}\right) \sum_{j=n-2}^{n} \frac{\delta F_{j}}{\delta w_{n-1}}=-c_{(n-1) n}+\eta^{2} c_{(n-1) n} \\
& \left(\frac{\partial}{\partial w_{n-1}}+\frac{\partial}{\partial w_{(n-1) x x}}\right) \sum_{j=n-1}^{n} \frac{\delta F_{j}}{\delta w_{n}}=-c_{(n-1) n}+\eta^{2} c_{(n-1) n}
\end{aligned}
$$

Having (18)-(19), (20)-(21), and (22)-(23) with the same right-hand sides ensures that the variational principle can be derived for the present problem. From (17), it follows that

$$
\begin{aligned}
& F_{1}\left(w_{1}, w_{2}\right)= \frac{c_{12}}{4} \Delta w_{21}^{2}+\frac{c_{12}}{4} \eta^{2}\left(\frac{\partial \Delta w_{21}}{\partial x}\right)^{2} \\
& F_{i}\left(w_{i-1}, w_{i}, w_{i+1}\right)= \frac{c_{(i-1) i}}{4} \Delta w_{i(i-1)}^{2}+\frac{c_{i(i+1)}}{4} \Delta w_{(i+1) i}^{2} \\
&+\frac{\eta^{2} c_{(i-1) i}}{4}\left(\frac{\partial \Delta w_{i(i-1)}}{\partial x}\right)^{2} \\
&+\frac{\eta^{2} c_{i(i+1)}}{4}\left(\frac{\partial \Delta w_{(i+1) i}}{\partial x}\right)^{2} \\
& \text { for } i=2,3, \ldots, n-1, \\
& F_{n}\left(w_{n-1}, w_{n}\right)= \frac{c_{(n-1) n}}{4} \Delta w_{n(n-1)}^{2}+\frac{\eta^{2} c_{(n-1) n}}{4}\left(\frac{\partial \Delta w_{n(n-1)}}{\partial x}\right)^{2} .
\end{aligned}
$$

With $F_{i}, i=1,2, \ldots, n$ given by (24), we observe that (15) are equivalent to (2)-(9).

\section{Hamilton's Principle}

The Hamilton' principle can be expressed as

$$
\int_{t_{1}}^{t_{2}}\left(\delta \mathrm{KE}(t)-\left(\delta W_{E}(t)+\delta \mathrm{PE}_{1}(t)+\delta \mathrm{PE}_{2}(t)\right)\right) d t=0,
$$

where

$$
\begin{aligned}
\mathrm{KE}(t)=\frac{1}{2} \sum_{i=1}^{i=n} \int_{0}^{L}( & \rho A_{i}\left(\frac{\partial w_{i}}{\partial t}\right)^{2}+\eta^{2} \rho A_{i}\left(\frac{\partial^{2} w_{i}}{\partial x \partial t}\right)^{2} \\
& \left.-\rho I_{i}\left(\frac{\partial \varphi_{i}}{\partial t}\right)^{2}+\eta^{2} \rho I_{i}\left(\frac{\partial^{2} \varphi_{i}}{\partial t \partial x}\right)^{2}\right) d x
\end{aligned}
$$




$$
\begin{aligned}
& W_{E}(t)=\frac{1}{2} \sum_{i=1}^{i=n} \int_{0}^{L}\left(-A_{i} \sigma_{x}\left(\frac{\partial w_{i}}{\partial x}\right)^{2}-\eta^{2} A_{i} \sigma_{x}\left(\frac{\partial^{2} w_{i}}{\partial x^{2}}\right)^{2}\right. \\
& \left.-f(x, t) w_{n}(x, t)\right) d x \\
& \mathrm{PE}_{1}(t)=\frac{1}{2} \sum_{i=1}^{i=n} \int_{0}^{L}\left(\kappa G A_{i}\left(\varphi_{i}-\frac{\partial w_{i}}{\partial x}\right)^{2}+E I_{i}\left(\frac{\partial \varphi_{i}}{\partial x}\right)^{2}+k w_{n}^{2}\right. \\
& \left.+k \eta^{2}\left(\frac{\partial w_{n}}{\partial x}\right)^{2}\right) d x \\
& \mathrm{PE}_{2}(t)=\frac{1}{2} \sum_{i=1}^{i=n} \int_{0}^{L}\left(c_{(i-1) i}\left(w_{i}-w_{i-1}\right)^{2}\right. \\
& \left.+\eta^{2} c_{(i-1) i}\left(\frac{\partial w_{i}}{\partial x}-\frac{\partial w_{i-1}}{\partial x}\right)^{2}\right) d x
\end{aligned}
$$

In (25)-(26), KE is the kinetic energy, $W_{E}$ is the work done by external forces, $\mathrm{PE}_{1}$ is the potential energy of deformation and $\mathrm{PE}_{2}$ is the potential energy due to van der Waals forces between the nanotubes.

\section{Boundary Conditions}

Next the variations of the functional $V\left(w_{i}, \varphi_{i}\right)$ in (12) are evaluated with respect to $w_{i}$ and $\varphi_{i}$ in order to derive the natural and geometric boundary conditions. Let $\delta w_{i}$ and $\delta \varphi_{i}$ denote the variations of $w_{i}$ and $\varphi_{i}$ such that $\delta w_{i}\left(x, t_{1}\right)=$ $\delta w_{i}\left(x, t_{2}\right)=\delta \varphi_{i}\left(x, t_{1}\right)=\delta \varphi_{i}\left(x, t_{2}\right)=0$. The first variations of $V\left(w_{i}, \varphi_{i}\right)$ with respect to $w_{i}$ and $\varphi_{i}$, denoted by $\delta_{w_{i}} V$ and $\delta_{\varphi_{i}} V$, respectively, can be obtained by integration by parts and expressed as

$$
\begin{aligned}
\delta_{w_{1}} V= & \delta_{w_{1}} V_{1}+\delta_{w_{1}} V_{2} \\
= & \int_{t_{1}}^{t_{2}} \int_{0}^{L} D_{a 1}\left(w_{1}, \varphi_{1}, w_{2}\right) \delta w_{1} d x d t+\partial \Omega_{a 1}(0, L, t), \\
\delta_{\varphi_{1}} V= & \delta_{\varphi_{1}} V_{1}=\int_{t_{1}}^{t_{2}} \int_{0}^{L} D_{b 1}\left(w_{1}, \varphi_{1}\right) \delta \varphi_{1} d x d t+\partial \Omega_{b 1}(0, L, t), \\
\delta_{w_{i}} V= & \sum_{j=i-1}^{i+1} \delta_{w_{i}} V_{j}=\int_{t_{1}}^{t_{2}} \int_{0}^{L} D_{a i}\left(w_{i-1}, w_{i}, \varphi_{i}, w_{i+1}\right) \delta w_{i} d x d t \\
& +\partial \Omega_{a i}(0, L, t) \quad \text { for } i=2, \ldots, n-1, \\
\delta_{\varphi_{i}} V= & \delta_{\varphi_{i}} V_{i}=\int_{t_{1}}^{t_{2}} \int_{0}^{L} D_{b i}\left(w_{i}, \varphi_{i}\right) \delta \varphi_{i} d x d t \\
& +\partial \Omega_{b i}(0, L, t) \text { for } i=2, \ldots, n-1, \\
\delta_{w_{n}} V= & \delta_{w_{n}} V_{n-1}+\delta_{w_{n}} V_{n}=\int_{t_{1}}^{t_{2}} \int_{0}^{L} D_{a n}\left(w_{n-1}, w_{n}, \varphi_{n}\right) \delta w_{n} d x d t \\
& +\partial \Omega_{a n}(0, L, t), \\
\delta_{\varphi_{n}} V= & \delta_{\varphi_{n}} V_{n}=\int_{t_{1}}^{t_{2}} \int_{0}^{L} D_{b n}\left(w_{n}, \varphi_{n}\right) \delta \varphi_{n} d x d t+\partial \Omega_{b n}(0, L, t), \\
& \\
&
\end{aligned}
$$

where $\partial \Omega_{i a}(0, L, t)$ and $\partial \Omega_{i b}(0, L, t)$ are the boundary terms defined as

$$
\begin{aligned}
& \partial \Omega_{a 1}(0, L, t) \\
& =-\left.A_{1} \sigma_{x} \eta^{2} \frac{\partial^{2} w_{1}}{\partial x^{2}} \delta w_{1}^{\prime}\right|_{x=0} ^{x=L}+\left.A_{1} \sigma_{x} \eta^{2} \frac{\partial^{3} w_{1}}{\partial x^{3}} \delta w_{1}\right|_{x=0} ^{x=L} \\
& +\left.\rho A_{1} \eta^{2} \frac{\partial^{3} w_{1}}{\partial x \partial t^{2}} \delta w_{1}\right|_{x=0} ^{x=L} \\
& +\left(-\kappa G A_{1}\left(\varphi_{1}-\frac{\partial w_{1}}{\partial x}\right)+\left(-A_{1} \sigma_{x}+\eta^{2} c_{12}\right) \frac{\partial w_{1}}{\partial x}\right. \\
& \left.\quad-\eta^{2} c_{12} \frac{\partial w_{2}}{\partial x}\right)\left.\delta w_{1}\right|_{x=0} ^{x=L},
\end{aligned}
$$

$\partial \Omega_{a i}(0, L, t)$

$$
\begin{aligned}
= & -\left.A_{i} \sigma_{x} \eta^{2} \frac{\partial^{2} w_{i}}{\partial x^{2}} \delta w_{1}^{\prime}\right|_{x=0} ^{x=L}+\left.A_{i} \sigma_{x} \eta^{2} \frac{\partial^{3} w_{i}}{\partial x^{3}} \delta w_{i}\right|_{x=0} ^{x=L} \\
+ & \left.\rho A_{i} \eta^{2} \frac{\partial^{3} w_{i}}{\partial x \partial t^{2}} \delta w_{i}\right|_{x=0} ^{x=L} \\
+ & {\left[-\kappa G A_{i}\left(\varphi_{i}-\frac{\partial w_{i}}{\partial x}\right)+\left(-A_{i} \sigma_{x}+\eta^{2}\left(c_{(i-1) i}+c_{i(i+1)}\right)\right)\right.} \\
& \left.\times \frac{\partial w_{i}}{\partial x}-\eta^{2}\left(c_{(i-1) i} \frac{\partial w_{i-1}}{\partial x}+c_{i(i+1)} \frac{\partial w_{i+1}}{\partial x}\right)\right]\left.\delta w_{i}\right|_{x=0} ^{x=L} \\
& \text { for } i=2,3, \ldots, n-1
\end{aligned}
$$

$\partial \Omega_{a n}(0, L, t)$

$$
\begin{aligned}
= & -\left.A_{n} \sigma_{x} \eta^{2} \frac{\partial^{2} w_{n}}{\partial x^{2}} \delta w_{n}^{\prime}\right|_{x=0} ^{x=L}+\left.A_{n} \sigma_{x} \eta^{2} \frac{\partial^{3} w_{n}}{\partial x^{3}} \delta w_{n}\right|_{x=0} ^{x=L} \\
+ & \left.\rho A_{n} \eta^{2} \frac{\partial^{3} w_{n}}{\partial x \partial t^{2}} \delta w_{n}\right|_{x=0} ^{x=L} \\
+ & {\left[-\kappa G A_{n}\left(\varphi_{n}-\frac{\partial w_{n}}{\partial x}\right)+\left(-A_{n} \sigma_{x}+\eta^{2}\left(c_{(n-1) n}+k\right)\right)\right.} \\
& \left.\times-\frac{\partial w_{n}}{\partial x} \eta^{2} c_{(n-1) n} \frac{\partial w_{n-1}}{\partial x}\right]\left.\delta w_{n}\right|_{x=0} ^{x=L}
\end{aligned}
$$

$\partial \Omega_{b i}(0, L, t)$

$$
=\left.\left(E I_{i} \frac{\partial \varphi_{i}}{\partial x}+\rho I_{i} \eta^{2} \frac{\partial^{3} \varphi_{i}}{\partial x \partial t^{2}}\right) \delta \varphi_{i}\right|_{x=0} ^{x=L} \text { for } i=1,2, \ldots, n,
$$

where $\delta w_{i}^{\prime}$ is the derivative of $\delta w_{i}$ with respect to $x$. Thus the boundary conditions at $x=0, L$ are given by

$$
\begin{array}{r}
E I_{i} \frac{\partial \varphi_{i}}{\partial x}+\rho I_{i} \eta^{2} \frac{\partial^{3} \varphi_{i}}{\partial x \partial t^{2}}=0 \text { or } \varphi_{i}=0 \\
\text { for } i=1,2, \ldots, n
\end{array}
$$




$$
\begin{aligned}
& \left(-A_{i} \sigma_{x} \eta^{2}\right) \frac{\partial^{2} w_{i}}{\partial x^{2}}=0 \quad \text { or } \quad \frac{\partial w_{i}}{\partial x}=0 \\
& \text { for } \sigma_{x} \neq 0, i=1,2, \ldots, n \text {, } \\
& A_{1} \sigma_{x} \eta^{2} \frac{\partial^{3} w_{1}}{\partial x^{3}}+\rho A_{1} \eta^{2} \frac{\partial^{3} w_{1}}{\partial x \partial t^{2}}-\kappa G A_{1}\left(\varphi_{1}-\frac{\partial w_{1}}{\partial x}\right) \\
& +\left(-A_{1} \sigma_{x}+\eta^{2} c_{12}\right) \frac{\partial w_{1}}{\partial x} \\
& -\eta^{2} c_{12} \frac{\partial w_{2}}{\partial x}=0 \quad \text { or } \quad w_{1}=0 \\
& A_{i} \sigma_{x} \eta^{2} \frac{\partial^{3} w_{i}}{\partial x^{3}}+\rho A_{i} \eta^{2} \frac{\partial^{3} w_{i}}{\partial x \partial t^{2}}-\kappa G A_{i}\left(\varphi_{i}-\frac{\partial w_{i}}{\partial x}\right) \\
& +\left(-A_{i} \sigma_{x}+\eta^{2}\left(c_{(i-1) i}+c_{i(i+1)}\right)\right) \frac{\partial w_{i}}{\partial x} \\
& -\eta^{2}\left(c_{(i-1) i} \frac{\partial w_{i-1}}{\partial x}+c_{i(+1)} \frac{\partial w_{i+1}}{\partial x}\right)=0 \\
& \text { or } \quad w_{i}=0 \text { for } i=2, \ldots, n-1 \\
& A_{n} \sigma_{x} \eta^{2} \frac{\partial^{3} w_{n}}{\partial x^{3}}+\rho A_{n} \eta^{2} \frac{\partial^{3} w_{n}}{\partial x \partial t^{2}}-\kappa G A_{n}\left(\varphi_{n}-\frac{\partial w_{n}}{\partial x}\right) \\
& +\left(-A_{n} \sigma_{x}+\eta^{2}\left(c_{(n-1) n}+k\right)\right) \frac{\partial w_{n}}{\partial x} \\
& -\eta^{2} c_{(n-1) n} \frac{\partial w_{n-1}}{\partial x}=0 \quad \text { or } \quad w_{n}=0 .
\end{aligned}
$$

Note that for $\sigma_{x}=0$, the boundary condition (30) is not needed. It is observed that for the small scale parameter $\eta>0$ (nonlocal theory) the natural boundary conditions are coupled and time derivative appears in the boundary conditions. These boundary conditions uncouple for $\eta=0$ (local theory) and time derivatives drop out.

\section{Conclusions}

Variational principles are derived using a semi-inverse variational method for multiwalled CNTs undergoing vibrations and modeled as nonlocal Timoshenko beams. Variational formulation of the problem facilitates the implementation of a number of computational approaches which, in most cases, simplify the method of solution as compared to the solution of a system of $2 n$ differential equations. The nonlocal elasticity theory accounts for small scale effects applicable to nanosized objects and Timoshenko beam model takes shear deformation into account which is not negligible in the case of nanotubes with small length-to-diameter ratio. As such they provide a more accurate model as compared to the Euler-Bernoulli model in the case of short nanotubes as pointed out in the papers $[9,10,23-25]$. The corresponding Hamilton's principle as well as the natural and geometric boundary conditions are derived. It is observed that the natural boundary conditions are coupled at the free end due to small scale effects being taken into account. The integrability conditions are also obtained which indicate whether a variational principle in the classical sense exists for the system of differential equations governing the vibrations of multiwalled nanotubes.

\section{References}

[1] S. Adali, "Variational principles for transversely vibrating multiwalled carbon nanotubes based on nonlocal eulerbernoulli beam model," Nano Letters, vol. 9, no. 5, pp. 17371741, 2009.

[2] B. I. Yakobson and R. E. Smalley, "Fullerene nanotubes: C1,000,000 and beyond," American Scientist, vol. 85, no. 4, pp. 324-337, 1997.

[3] V. M. Harik, "Ranges of applicability for the continuum beam model in the mechanics of carbon nanotubes and nanorods," Solid State Communications, vol. 120, no. 7-8, pp. 331-335, 2001.

[4] C. Q. Ru, "Column buckling of multiwalled carbon nanotubes with interlayer radial displacements," Physical Review B, vol. 62, no. 24, pp. 16962-16967, 2000.

[5] Q. Wang and V. K. Varadan, "Stability analysis of carbon nanotubes via continuum models," Smart Materials and Structures, vol. 14, no. 1, pp. 281-286, 2005.

[6] Q. Wang, T. Hu, G. Chen, and Q. Jiang, "Bending instability characteristics of double-walled carbon nanotubes," Physical Review B, vol. 71, no. 4, Article ID :045403, 8 pages, 2005.

[7] A. Sears and R. C. Batra, "Buckling of multiwalled carbon nanotubes under axial compression," Physical Review B, vol. 73, no. 8, Article ID 085410, 11 pages, 2006.

[8] Y. Q. Zhang, X. Liu, and J. H. Zhao, "Influence of temperature change on column buckling of multiwalled carbon nanotubes," Physics Letters A, vol. 372, no. 10, pp. 1676-1681, 2008.

[9] Y. Y. Zhang, C. M. Wang, and V. B. C. Tan, "Buckling of multiwalled carbon nanotubes using Timoshenko beam theory," Journal of Engineering Mechanics, vol. 132, no. 9, pp. 952-958, 2006.

[10] C. M. Wang, V. B. C. Tan, and Y. Y. Zhang, "Timoshenko beam model for vibration analysis of multi-walled carbon nanotubes," Journal of Sound and Vibration, vol. 294, no. 4, pp. 1060-1072, 2006.

[11] R. E. Miller and V. B. Shenoy, "Size-dependent elastic properties of nanosized structural elements," Nanotechnology, vol. 11, no. 3, pp. 139-147, 2000.

[12] T. Chang and H. Gao, "Size-dependent elastic properties of a single-walled carbon nanotube via a molecular mechanics model," Journal of the Mechanics and Physics of Solids, vol. 51, no. 6, pp. 1059-1074, 2003.

[13] C. T. Sun and H. Zhang, "Size-dependent elastic moduli of platelike nanomaterials," Journal of Applied Physics, vol. 93, no. 2, pp. 1212-1218, 2003.

[14] C. W. Lim and L. H. He, "Size-dependent nonlinear response of thin elastic films with nano-scale thickness," International Journal of Mechanical Sciences, vol. 46, no. 11, pp. 1715-1726, 2004.

[15] D. W. Huang, "Size-dependent response of ultra-thin films with surface effects," International Journal of Solids and Structures, vol. 45, no. 2, pp. 568-579, 2008.

[16] D. G. B. Edelen and N. Laws, "On the thermodynamics of systems with nonlocality," Archive for Rational Mechanics and Analysis, vol. 43, no. 1, pp. 24-35, 1971.

[17] A. C. Eringen, "Linear theory of nonlocal elasticity and dispersion of plane waves," International Journal of Engineering Science, vol. 10, no. 5, pp. 425-435, 1972. 
[18] J. Peddieson, G. R. Buchanan, and R. P. McNitt, "Application of nonlocal continuum models to nanotechnology," International Journal of Engineering Science, vol. 41, no. 3-5, pp. 305312, 2003.

[19] M. Xu, "Free transverse vibrations of nano-to-micron scale beams," Proceedings of the Royal Society A, vol. 462, no. 2074, pp. 2977-2995, 2006.

[20] C. M. Wang, Y. Y. Zhang, S. S. Ramesh, and S. Kitipornchai, "Buckling analysis of micro- and nano-rods/tubes based on nonlocal Timoshenko beam theory," Journal of Physics D, vol. 39, no. 17, pp. 3904-3909, 2006.

[21] Q. Wang and Y. Shindo, "Nonlocal continuum models for carbon nanotubes subjected to static loading," Journal of Mechanics of Materials and Structures, vol. 1, no. 4, pp. 663680, 2006.

[22] Q. Wang and K. M. Liew, "Application of nonlocal continuum mechanics to static analysis of micro- and nano-structures," Physics Letters A, vol. 363, no. 3, pp. 236-242, 2007.

[23] J. N. Reddy, "Nonlocal theories for bending, buckling and vibration of beams," International Journal of Engineering Science, vol. 45, no. 2-8, pp. 288-307, 2007.

[24] C. M. Wang, Y. Y. Zhang, and X. Q. He, "Vibration of nonlocal Timoshenko beams," Nanotechnology, vol. 18, no. 10, Article ID 105401, 9 pages, 2007.

[25] C. M. Wang, S. Kitipornchai, C. W. Lim, and M. Eisenberger, "Beam bending solutions based on nonlocal Timoshenko beam theory," Journal of Engineering Mechanics, vol. 134, no. 6, pp. 475-481, 2008.

[26] R. Artan and A. Tepe, "The initial values method for buckling of nonlocal bars with application in nanotechnology," European Journal of Mechanics A, vol. 27, no. 3, pp. 469-477, 2008.

[27] J.-C. Hsu, R.-P. Chang, and W.-J. Chang, "Resonance frequency of chiral single-walled carbon nanotubes using Timoshenko beam theory," Physics Letters A, vol. 372, no. 16, pp. 2757-2759, 2008.

[28] L. J. Sudak, "Column buckling of multiwalled carbon nanotubes using nonlocal continuum mechanics," Journal of Applied Physics, vol. 94, no. 11, pp. 7281-7287, 2003.

[29] Q. Wang, "Wave propagation in carbon nanotubes via nonlocal continuum mechanics," Journal of Applied Physics, vol. 98, no. 12, Article ID 124301, 6 pages, 2005.

[30] L. Wang and H. Hu, "Flexural wave propagation in singlewalled carbon nanotubes," Physical Review B, vol. 71, no. 19, Article ID 195412, 7 pages, 2005.

[31] Q. Wang, G. Y. Zhou, and K. C. Lin, "Scale effect on wave propagation of double-walled carbon nanotubes," International Journal of Solids and Structures, vol. 43, no. 20, pp. 60716084, 2006.

[32] P. Lu, H. P. Lee, C. Lu, and P. Q. Zhang, "Application of nonlocal beam models for carbon nanotubes," International Journal of Solids and Structures, vol. 44, no. 16, pp. 5289-5300, 2007.

[33] H. Heireche, A. Tounsi, A. Benzair, M. Maachou, and E. A. Adda Bedia, "Sound wave propagation in single-walled carbon nanotubes using nonlocal elasticity," Physica E, vol. 40, no. 8, pp. 2791-2799, 2008.

[34] S. Adali, "Variational principles for multi-walled carbon nanotubes undergoing buckling based on nonlocal elasticity theory," Physics Letters A, vol. 372, no. 35, pp. 5701-5705, 2008.

[35] S. Adali, "Variational principles for multi-walled carbon nanotubes undergoing nonlinear vibrations by semi-inverse method," Micro and Nano Letters, vol. 4, no. 4, pp. 198-203, 2009.
[36] J.-H. He, "Semi-inverse method of establishing generalized variational principles for fluid mechanics with emphasis on turbomachinery aerodynamics," International Journal of Turbo and Jet Engines, vol. 14, no. 1, pp. 23-28, 1997.

[37] J.-H. He, "Variational principles for some nonlinear partial differential equations with variable coefficients," Chaos, Solitons and Fractals, vol. 19, no. 4, pp. 847-851, 2004.

[38] J.-H. He, "Variational approach to $(2+1)$-dimensional dispersive long water equations," Physics Letters A, vol. 335, no. 2-3, pp. 182-184, 2005.

[39] H.-M. Liu, "Generalized variational principles for ion acoustic plasma waves by He's semi-inverse method," Chaos, Solitons and Fractals, vol. 23, no. 2, pp. 573-576, 2005.

[40] J.-H. He, "Variational theory for one-dimensional longitudinal beam dynamics," Physics Letters A, vol. 352, no. 4-5, pp. 276-277, 2006.

[41] X.-W. Zhou, "Variational approach to the Broer-KaupKupershmidt equation," Physics Letters A, vol. 363, no. 1-2, pp. 108-109, 2007.

[42] J.-H. He, "Variational principle for two-dimensional incompressible inviscid flow," Physics Letters A, vol. 371, no. 1-2, pp. 39-40, 2007. 

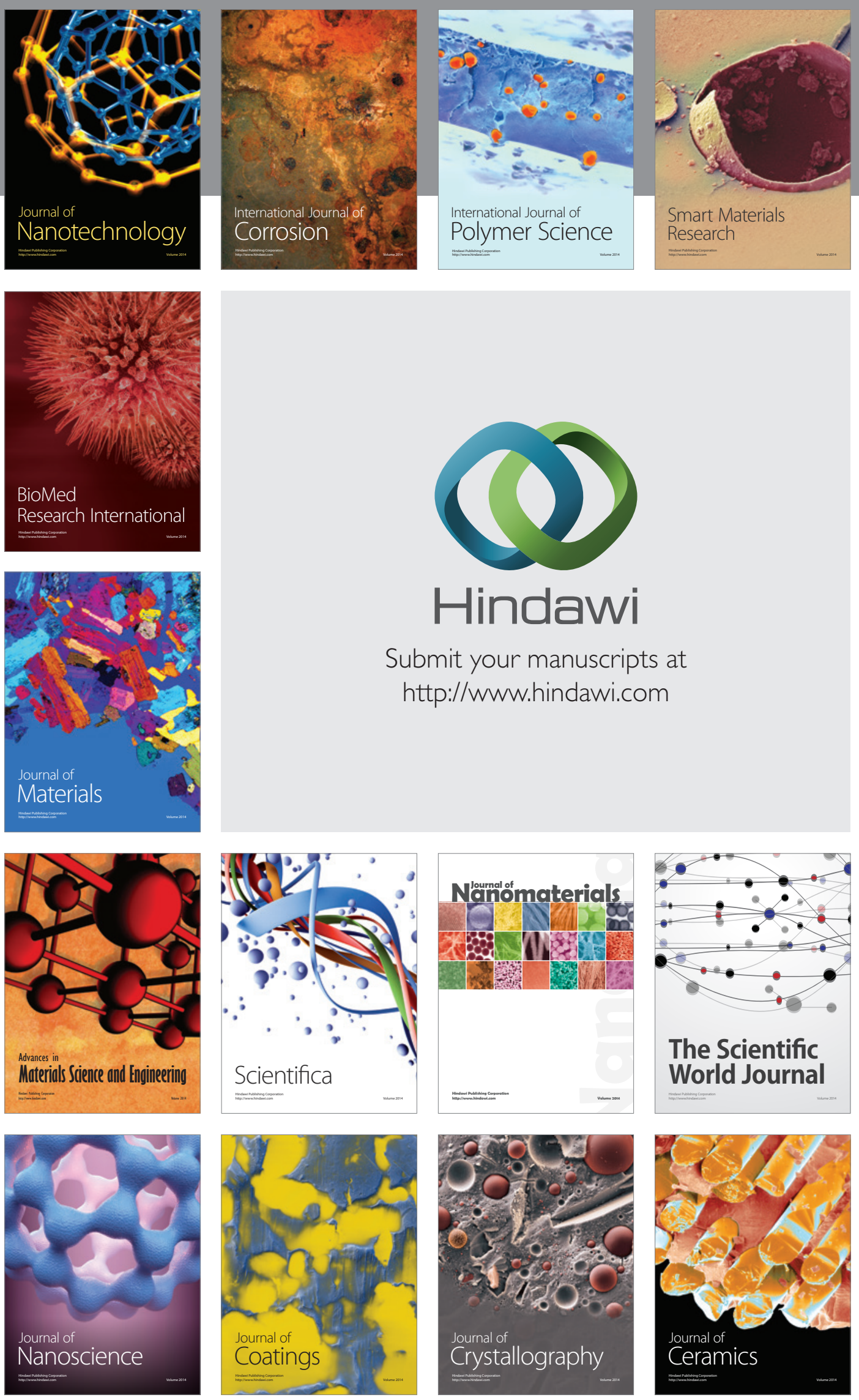

The Scientific World Journal

Submit your manuscripts at

http://www.hindawi.com

\section{World Journal}

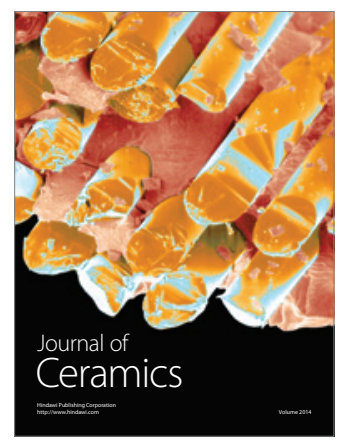

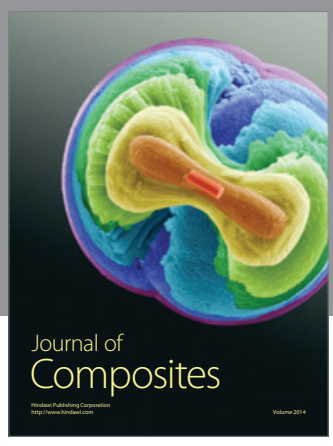
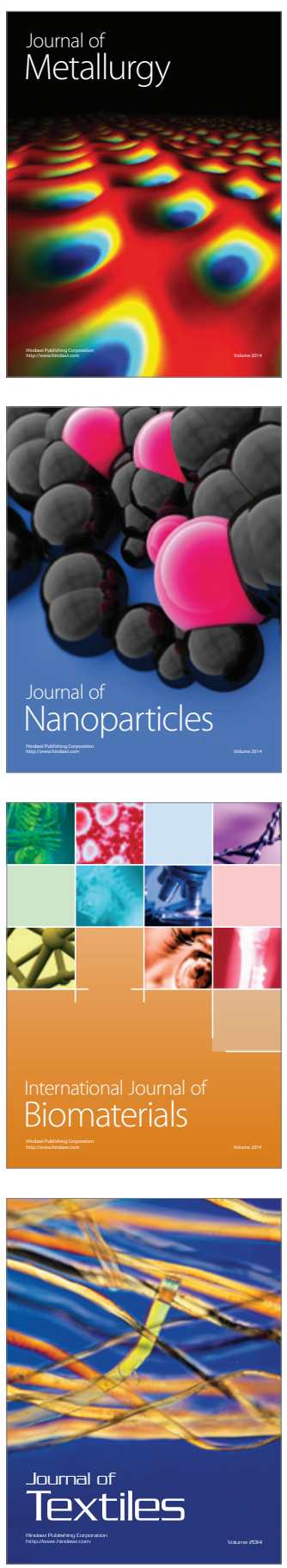